\title{
An Across-Store Analysis of Intrinsic and Extrinsic Cross-Category Effects
}

\author{
Venkatesh Shankar • P. K. Kannan
}

Published online: 18 April 2014

(C) Springer Science+Business Media New York 2014

\begin{abstract}
An important part of the rapidly growing shopper marketing practice is cross-category retail management. In managing two related product categories, retailers face some important questions: Which category should be stocked more? How close to each other should they be stocked in the store (aisle adjacency)? Which category should be promoted more often? And when should the two categories be sold as a bundle? To address these questions, we examine how purchases of related product and subproduct categories influence one another, and how the relative aisle locations of two related product categories influence their respective purchases. We consider both extrinsic (aisle location-based) and intrinsic (affinity-based) cross-category effects. Using aggregate store level data together with store descriptor and store shopper demographic data, we estimate a simultaneous system of models for two related product categories, soft drinks and salty snacks. We also estimate a system of salty snack subcategory purchase models. We find that both extrinsic and intrinsic cross-category effects are asymmetric, that is, different categories and subcategories have different effects on one another. We discuss the theoretical and managerial implications of these findings.
\end{abstract}

Keywords Shopper marketing · Category management . Retailing $\cdot$ Product management $\cdot$ Merchandising · Econometrics

\footnotetext{
V. Shankar $(\bowtie)$

Center for Retailing Studies, Mays Business School, Texas A\&M

University, College Station, USA

e-mail: VShankar@mays.tamu.edu

\section{P. K. Kannan}

Robert H. Smith School of Business, University of Maryland, College Park, USA

e-mail: pkannan@rhsmith.umd.edu
}

\section{Introduction}

Shopper marketing, the planning and execution of all marketing activities that influence a shopper along - and beyond - the path-to-purchase, from shopping trigger to purchase, consumption, repurchase, and recommendation stages [28, 29, 31] is on the ascendancy. An important part of the rapidly growing shopper marketing practice is cross-category retail management. Product category interrelationships raise a number of critical but often hard-to-resolve issues for retailers. Consider a typical US grocery store that carries approximately 31,000 items in roughly 600 product categories. Such product diversity presents retailers with the complex task of managing multiple product categories, such as soft drinks and salty snacks, or subcategories, such as potato, tortilla, and corn chips within the salty snack category. In particular, the formulation of effective merchandising strategies across categories is important [5, 10, 23]. Store profitability is maximized when consumers purchase across categories [17]. Furthermore, retailers' promotion pass through decisions are based on cross-category demand relationships [22].

Developing effective cross-category strategies requires that retailers answer several questions: Which product category should be stocked more or less? ${ }^{1}$ Where should they be physically located in the store, and in particular, should they be placed on separate or adjacent aisles? Which product category should be promoted more often? When should the product categories be bundled or sold separately? To answer such questions, store managers need to identify demand interrelationships across product and subproduct categories, and assess the impact of one category's within-store location on the sales of another. The selections should capitalize on crosscategory demand interrelationships.

\footnotetext{
${ }^{1}$ Retailers also need to address the issue of how much shelf space to allocate to each brand. Since our paper does not consider shelf space issues, we do not pursue this issue in the paper.
} 
To study cross-category relationships, market basket data collected from a panel of shoppers are used (e.g., [2, 4, 18, 25, 27]). However, the availability and use of such data offer important challenges for managers. First, the data needs are often large. Analysis of the purchases of many consumers over time and across several categories for a reasonable set of measures requires intensive and expensive data collection efforts and storage. Second, the collection and analysis of market basket data for multiple categories is often expensive and burdensome because there are $2^{\mathrm{n}}-1$ theoretically possible category combinations involving $\mathrm{n}$ categories [27]. Finally, to be useful for weekly decision-making, market basket data should allow for the analysis of changes in store level variables.

Since such an analysis is complex, managers seldom perform category analysis of market basket panel data. While modern computers make handling such volumes of data relatively easy, many store managers may prefer to work with more readily available data using simpler and more accessible approaches. Frequently, they have access to data at the aggregate store level, where such data can often be readily obtained and analyzed. In sum, while market basket data are preferred for a consumer level understanding of cross-category relationships, practical concerns often lead many store managers to consider alternative approaches.

We propose that aggregate, across-store data provide useful and important insights into cross-category relationships. First, chain or store level data analysis has been shown to offer important managerial insights into own and cross brand price elasticities $[6,30,35]$. Such data minimize the many complexities and provides actionable results at the chain or store level.

Second, analysis of chain or store level aggregate data over long periods (say 1 year) is computationally less intensive than weekly data. For example, in the application subsequently described in this paper that is based on aggregate data over a 2-year time period, the data needs fall to fewer than 500 observations. Furthermore, because many grocery chains have consistent pricing and promotions across their stores, by analyzing aggregate data, we can control for the promotion effects on category purchases and focus on intrinsic and extrinsic cross-category effects.

Finally, because products' aisle locations typically do not change much over time, market basket data do not let managers study the effects of one aisle location relative to another. Cross-sectional aggregate data, on the other hand, can help analyze the associations between relative aisle location and purchases in multiple categories [5]. In sum, cross-sectional aggregate data across different stores offer a number of important benefits to retail store managers, and could serve as a useful complement to analysis of market basket panel data.

In this paper, we propose a conceptual framework of crosscategory effects and advance key hypotheses that underlie this framework. We test these hypotheses using an empirical approach designed to answer the four questions raised earlier using aggregate, across-store data on two product categories (soft drinks and salty snacks), and on multiple subcategories within one category (salty snacks). Soft drinks and salty snacks are important categories for retailers (e.g., $[5,8])$. We demonstrate that cross-category intrinsic and extrinsic, and aisle adjacency effects, can be meaningfully analyzed with such data. Using a simultaneous system of models, we show how managers can readily perform an empirical analysis of cross-category effects using average store transaction, store descriptor, and store shopper demographic data.

Our paper differs from and extends prior research on crosscategory effects. It adds to the multicategory analysis literature (e.g., $[5,11,18,27])$, by examining the effects of subcategories on one another and the role of aisle adjacency across categories and subcategories. In particular, it differs from Bezawada et al. [5], who also study the effects of aisle adjacency of categories, in key respects. First, while Bezawada et al. [5] examine aisle adjacency and display effects, we address the overall cross-category effect. Second, unlike Bezawada et al. [5], we analyze the effects of subproduct categories on one another. Third, while Bezawada et al. [5] use a spatial model, we use a simultaneous equation model of sales. Fourth, Bezawada et al. [5] use store level unit sales data, whereas we use transactions data. Thus, our research extends Bezawada et al. [5] and the category management, shopper marketing, and retailing literatures.

Our empirical results show that both the intrinsic and the extrinsic effects of salty snacks and of soft drinks on each other are asymmetric. Specifically, greater purchases of soft drinks lead to greater purchases of salty snacks, but the converse is not true, and the adjacency of salty snacks and soft drinks benefit purchases of salty snacks, but does not lift purchases of soft drinks. Furthermore, some subcategories of salty snacks, such as potato chips, tortilla chips, corn chips, and popcorn, are intrinsic substitutes, but each of these subcategories is intrinsically complementary with other subcategories such as cheese puffs and pretzels.

Based on our results, we propose a managerial framework designed to help retailers approach the cross-category relationships problem. In the next section, we present the conceptual background and hypotheses in our study. Then, we formulate the models and describe how we estimate their parameters. Finally, we discuss the managerial implications of our findings and propose a general framework for managing demand across product categories and subcategories.

\section{Conceptual Background and Hypotheses}

\subsection{Intrinsic and Extrinsic Demand Relationships}

The demand for products in one category is often related to that in another category $[5,11,13,21,26]$. Conceptually, two 
types of cross-category effects exist, intrinsic and extrinsic. Two product categories are intrinsically related if the purchase of a product in one category influences the purchase of a product in another category based on affinity in consumption. Affinity in consumption exists between two categories if the consumption of one category typically triggers thoughts about the other category. For example, the consumption of pasta might prompt thoughts of consuming pasta sauce, cheese, and beverages. Similarly, salty snack consumption could spur the thought of consuming a soft drink and vice versa. In some cases, the consumption of one category may deter the consumption of another category, leading to a negative affinity between the two categories. For example, the consumption of a chilled beverage such as cola may dissuade the consumption of a hot beverage like coffee during that consumption occasion or moment. Similarly, ice cream and nonfat yogurt may have negative affinities. Typically, ice cream is viewed as a treat, whereas nonfat yogurt is perceived as a healthier alternative. Therefore, categories can have either positive or negative affinities toward each other based on shoppers' prior purchase, consumption experience, and consumption occasion.

Two product categories are extrinsically related if temporal factors, such as price changes and promotional activities, and nontemporal factors, such as relative product locations within the store, associated with one category influence the purchases of another category. Sometimes, a promotion in one category can trigger the purchase of items in another category, leading to positive extrinsic relationship between the two categories. For example, a promotion on toothpaste may induce the purchases of multiple packages, which in turn could lead to purchases of mouthwash or toothbrush. In other cases, a promotion in one category might result in reduced purchases of another category, yielding a negative extrinsic relationship between the two categories. For example, a promotion in the coffee category might lead to lower purchases in the tea category.

The theoretical basis for intrinsic and extrinsic crosscategory effects lies in the memory literature. Shoppers have organized associative networks of product categories in memory [20,32]. Parts of this knowledge are cued and retrieved from memory. ${ }^{2}$ Through a process of spreading activation [3], the retrieval of this category (e.g., soft drinks) knowledge leads to the activation and retrieval of knowledge in related product categories (e.g., salty snacks). Therefore, both intrinsic and extrinsic cross-category effects will depend upon shoppers' category knowledge, but the nature of the initiating cues may be different.

With intrinsic effects, merely encountering in store a product from one category cues the retrieval of knowledge in

\footnotetext{
${ }^{2}$ For simplicity, in this discussion, we ignore the processes by which shoppers classify products into categories, see Cohen and Basu [7].
}

related categories. For example, such encounters might conjure up mental images in shoppers' minds of consuming related products, leading to the purchase of complementary categories. In-store product cueing may also remind the shopper of unmet needs [15] or product substitutes from other, related product categories, spurring their purchases.

The extrinsic effect works similarly, except that the shopper's associative network is cued by marketing activities designed to influence product purchase. Some marketing actions, such as deals, feature advertisements, and in-store endof-aisle displays, are temporal [1]. Others, such as aisle adjacency or the placement of two product categories in close proximity to one another in the store, typically last longer.

Both intrinsic and extrinsic cross-category purchase effects may be either positive or negative, making the categories in question complements or substitutes, respectively. When the intrinsic or extrinsic relationship between two categories is positive, we term the categories, complementary categories. By the same token, if the relationship is negative, we call the categories, substitute categories.

Goal theory suggests that consumers will likely buy categories that will fulfill their goals. Complementary categories are purchased in anticipation of their joint consumption and use toward a common goal (e.g., toothpaste and toothbrush for oral hygiene, salty snacks and soft drinks for a light meal), whereas substitute categories are purchased as replacements for the same consumption goal (e.g., diet cola and regular cola, or tortilla or potato chips for use with dips) [24]. Thus, substitution reflects shoppers' perceptions that products have a similar form or functionality. Our view of complementarity differs somewhat from that of Manchanda et al. [18], who define complementarity as existing when marketing activity in one category influences purchases of another category (extrinsic effect). However, they use category coincidence to refer to situations where all reasons (except category complementarity and consumer heterogeneity) induce a joint purchase of two or more categories (cf. intrinsic effect).

\subsection{Extrinsic Effects of Aisle Adjacency}

Aisle adjacency is an important source of extrinsic crosscategory effects. At a conceptual level, an adjacency spectrum of in-store product placement options can be defined by their proximity. At one extreme are bundled products, which are placed and sold together, i.e., proximity is very high. ${ }^{3}$ Shelf location adjacency reduces the level of proximity by allowing for products to be placed on shelves where their respective positions vary by eye level and location centrality on a given

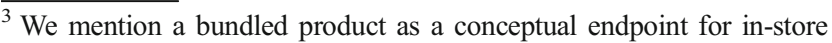
product proximity. However, this research focuses on the proximity of different categories' locations within the store. Because its relevance to this paper is peripheral, we do not review bundling research.
} 
set of shelves [1]. Consistent with Bezawada et al. [5], we define aisle adjacency between two categories as the proximity between the product categories' shelf locations; it allows for less proximity where two product categories are placed side by side on the same side of an aisle, on opposite sides of the aisle, or on adjacent aisles. Finally, the lowest level of proximity occurs when products are displayed in aisle locations that are widely separated in the store. Aisle adjacency for two product categories can range from very high to very low.

Product placement decisions have important implications for stores' sales and costs $[5,10]$. Previous research has examined the topic, but its emphasis has varied considerably across the adjacency spectrum. A great deal of academic research has focused on shelf space issues of store placement. For example, Urban [34] reviews over ten shelf placement studies before presenting a model that generalizes and integrates shelf space models with inventory control and product assortment models. However, studies focusing on aisle adjacency and its effects on products' sales have been limited [10]. Furthermore, reviewing the literature on product placement returns only a few studies; this may be due to a lack of availability of appropriate data. This lack of attention is surprising given the importance of the topic for retailers. For example, Jitney Jingle, a Jackson Mississippi-based chain, has "power alleys" that present products from related categories, such as snacks and beverages. The underlying rationale is convenience and ease of shopping. Similarly, Albertsons, one of the largest retail chains in the U.S., has a coordinated merchandising strategy for snacks and related categories.

Retailers' concern with aisle adjacency likely reflects their understanding of shopper behavior. Consider a shopper whose goal is to make lunches to take to work in the next week. This shopper's goals are represented by a set of needs that can be met by various product categories [16], some of which are complements (meat and cheese slices), whereas others are substitutes (juices and soft drinks). Increasing aisle adjacency between these product categories offers the shopper several benefits for attaining the lunch goal; specifically, it reduces information search complexity by making assortment assembly easier; it means less time walking around the store, enhancing convenience; it provides in-store cueing of forgotten needs; and it satisfies variety seeking by offering new product choice to meet the goal [24]. With shoppers increasingly focused on convenience, a move to goal-based in-store placement versus one that is functional or product category-based should enhance shopper satisfaction and loyalty [5].

Based on the previous reasoning, we propose the following hypotheses in the context of two complementary product categories, soft drinks and salty snacks, and subcategories of salty snacks, such as potato chips, tortilla chips, and pretzels:
Intrinsic Cross-category Effects

$\mathrm{H}_{1}$ : Greater purchases of one category will be associated with greater purchases of a complementary category, and vice versa.

$\mathrm{H}_{2}$ : Subcategories with (dis)similar form and function will exhibit purchase (complementarity) substitution.

\section{Extrinsic Cross-category Effects}

$\mathrm{H}_{3}$ : Aisle adjacency of two complementary categories will be positively associated with purchases of those categories.

\section{Data}

To test these hypotheses, we collected data from 34 stores belonging to an upstate New York supermarket chain. For each store in the chain, the transaction data contain the average proportion of transactions in which different subcategories of salty snacks and the soft drink category were bought over a 2year period. The supermarket chain provided the transaction data, aisle adjacency data, and the store descriptor data containing store size and the age of the store (date of each store's opening). Because geodemographics data drive category and retail purchases [14], we obtained the store shopper demographics data from Spectra Information Services and linked these data to the other data through the store variable.

\section{Model Formulation and Estimation}

\subsection{Model Formulation}

To study cross-category effects, we develop a simultaneous equation model comprising equations for the purchases of salty snacks and soft drinks. Similarly, to examine the relationships among the subcategories of salty snacks, we formulate a simultaneous equation model containing equations for the purchases of subcategories. In each model, our focal independent variables are purchases of related categories and subcategories, and aisle adjacencies. In addition, we control for the following other variables that may potentially influence shoppers' (sub) category purchases: (1) (sub) categoryspecific variables, such as shelf footage, (2) store descriptor variables, such as store size and age of the store, and (3) store shopper demographic variables, such as average age and average household income.

The purpose of our proposed models is to identify the cross-category and cross-subcategory effects that reveal complements and substitutes, and to explore the nature of intrinsic 
and extrinsic effects. The identification of similar groups of categories (substitutes or complements) can be achieved through other types of analyses, such as cluster analysis and decision trees. However, a simultaneous model analysis provides the signs of the coefficients of the category or subcategory purchases, which indicate whether the categories or subcategories are complements or substitutes, and whether the cross-category or cross-subcategory effects are symmetric or asymmetric, providing an important advantage. Such directional insights cannot be obtained from other approaches, such as cluster analysis.

\subsubsection{Cross-Category Effects}

The Category 1 (salty snack) purchases model is given by:

$$
\begin{aligned}
\operatorname{SSNACK}_{i}= & \alpha_{0}+\alpha_{1} \text { BEV }_{i}+\alpha_{2} \text { ADJ }_{i}+\alpha_{3} \text { SSFT }_{i} \\
& +\alpha_{4} \text { STRAGE }_{i}+\alpha_{5} \text { SSIZE }_{i}+\alpha_{6} \text { HHAGE }_{i} \\
& +\alpha_{7} \text { HHINC }_{i}+\varepsilon_{i}
\end{aligned}
$$

where SSNACK is the percentage of transactions with salty snacks purchase, $\mathrm{BEV}$ is the percentage of transactions with a soft drink beverage purchase, ADJ is the adjacency of the salty snacks aisle with the beverage aisle (measured by the number of aisles between the locations of the two categories in the store), SSFT is salty snack area in the store in square feet, STRAGE is the age of the store in terms of months since opening, SSIZE is the store size or average sales of the store over the time period of the data ( $\$$ weekly sales), HHAGE is the average household age, and HHINC is average household income ( $\$$ ' $000 \mathrm{~s}$ ), all for each store i. $\alpha_{0}$ to $\alpha_{7}$ are the associated parameters. Finally, $\varepsilon$ is an error-term assumed to be normally distributed with zero mean. Because our model is based on cross-sectional data across stores, we do not need to use time-varying variables, such as promotional variables, as explanatory variables. ${ }^{4}$

Similarly, the model for the complementary category 2 (soft drinks) is given by:

$$
\begin{aligned}
B E V_{i}= & \beta_{0}+\beta_{1} \operatorname{SSNACK}_{i}+\beta_{2} \text { ADJ }_{i}+\beta_{3} \text { BSFT }_{i} \\
& +\beta_{4} \text { STRAGE }_{i}+\beta_{5} \text { SSIZE }_{i}+\beta_{6} \text { HHAGE }_{i} \\
& +\beta_{7} \text { HHINC }_{i}+\eta_{i}
\end{aligned}
$$

\footnotetext{
${ }^{4}$ Many retail chains, including the chain we study, typically have common pricing and promotional variables across stores in a geographical area. Therefore, even if we were to include average pricing and promotion variables in our cross-sectional model, such variables will unlikely differ across stores within the same chain.
}

where BSFT is the soft drink beverage area in the store and $\beta_{0}$ to $\beta_{7}$ are the associated parameters. $\eta$ is an error-term assumed to be normally distributed with zero mean, and the other terms are as defined earlier.The coefficients $\alpha_{1}$ and $\beta_{1}$ capture the effects of soft drinks on salty snacks and vice versa, respectively. Hypothesis $\mathrm{H}_{1 \mathrm{a}}$ implies $\alpha_{1}>0$ and is significant, and $\mathrm{H}_{1 \mathrm{~b}}$ implies $\beta_{1}>0$ and is significant. The coefficients $\alpha_{2}$ and $\beta_{2}$ capture the effects of aisle adjacency of soft drinks and salty snacks on purchases of salty snacks and vice versa, respectively. According to Hypothesis $\mathrm{H}_{3 \mathrm{a}}, \alpha_{2}>0$ and is significant. According to $\mathrm{H}_{3 \mathrm{~b}}, \beta_{2}>0$ and is significant.

\subsubsection{Cross-Subcategory Effects}

The model for each subcategory purchases is given by:

$$
\begin{aligned}
S_{S A T_{j i}=} & \gamma_{0 j}+\gamma_{1 j} B E V_{i}+\gamma_{2 j} A D J_{j i}+\gamma_{3 j} \text { LINFT }_{j i} \\
& +\gamma_{4 j} S \operatorname{STAGE_{i}}+\gamma_{5 j L} S S I Z E_{i}+\gamma_{6 j} H H A G E_{i} \\
& +\gamma_{7 j} H_{H} H I N C_{i}+\sum_{l=1, l \neq j} \gamma_{8 j l} S C A T_{l i}+\varsigma_{j i}
\end{aligned}
$$

where SCAT is the percentage of transactions involving the purchase of the subcategory, LINFT is the linear footage of the subcategory in the store, $\mathrm{j}, \mathrm{l}=$ subcategory $\in$ (potato chips, tortilla chips, corn chips, cheese puffs, pop corn, pretzels), $\gamma_{0}$ to $\gamma_{7}$ are the associated parameters, $\varsigma$ is an error term assumed to be normally distributed with zero mean, and the other terms are as defined earlier. ${ }^{5}$

The coefficients $\gamma_{8 \mathrm{jl}}$ capture the effects of the purchases of subcategories on the purchases of other subcategories. Hypothesis $\mathrm{H}_{2}$ implies that some of these coefficients are positive and significant, whereas some others are negative and significant. ${ }^{6}$

\subsection{Model Estimation}

We estimate models (1), (2), and (3) using the three-stage least squares (3SLS) method, which captures the endogeneity of purchases of different categories and subcategories. In addition, 3SLS accounts for any correlation between the errorterms of the equations that could be due to common, omitted variables, i.e., Seemingly Unrelated Regression (SUR, [36]).

\footnotetext{
${ }^{5}$ Because each subcategory is in the same aisle as the parent category, we do not have the relative aisle adjacencies of the subcategories as independent variables. Instead, we use the aisle adjacency with respect to soft drinks as the independent variable.

${ }^{6}$ We could potentially add another model with aisle adjacency as a function of exogenous variables such as category shelf space requirement, category sales, and other in-store locational constraints. However data on these additional exogenous variables are not easy to obtain. Moreover, stores do not vary aisle adjacency systematically over time for us to obtain more observations.
} 
We estimate Eqs. (1) and (2) as one system of equations, and Eq. (3) as a separate system of equations across the subcategories.

\section{Results}

\subsection{Cross-Category Effects}

The results of the 3SLS models for salty snacks and soft drink beverage purchases (Eqs. 1 and 2) ${ }^{7}$ appear in Table 1. In the salty snacks model, the coefficient of BEV, representing the effect of soft drinks purchases, is positive and significant $(p<0.05)$, supporting $\mathrm{H}_{1 \mathrm{a}}$. Aisle adjacency level (ADJ) is also positive and significant $(p<0.05)$, consistent with $\mathrm{H}_{3 \mathrm{a}}$. The proportion of salty snack purchases is greater when beverage purchase proportion increases, and as the salty snacks aisle becomes more adjacent to the soft drinks aisle.

In addition, among the control variables, average household age, HHAGE $(p<0.01)$ and household income, HHINC $(p<0.05)$ are significant; salty snack purchases are greater in stores with higher average household age and household income.

However, the soft drinks model shows that purchases of salty snacks, and the adjacency of soft drinks and salty snacks, are not significant predictors of soft drink purchases, contrary to hypotheses $\mathrm{H}_{1 \mathrm{~b}}$ and $\mathrm{H}_{3 \mathrm{~b}}$, respectively. While salty snack purchases are enhanced by soft drink purchases and by the adjacency of salty snacks with soft drinks, the converse is not true. Thus, there are asymmetries in both the intrinsic and the extrinsic (aisle adjacency) cross-category effects.

These results, however, are consistent with the typical roles of the two product categories in most stores. Soft drinks are typically managed as a "destination" category that serves to draw consumers to the store [33]. Because it is a destination category, consumers visit a store to purchase beverages first. Therefore, the proportion of transactions involving beverage purchases is not significantly dependent on the proportion of transactions containing purchases of other categories, including salty snacks. By the same token, on many occasions, such as parties, light meals, and the like, purchases of soft drinks are likely to trigger purchases of salty snacks (an intrinsic cross-category effect). Interestingly, beverage purchases decrease with household income $(p<0.01)$, i.e., stores frequented by shoppers with higher average household income tend to have a lower proportion of soft drink purchases. Shoppers with higher incomes are likely to have higher

\footnotetext{
${ }^{7}$ We also estimated all the models using ordinary least squares (OLS). However, all models showed significant and high cross-error correlation between the salty snack and beverage equations. Therefore, we interpret the 3SLS results. The substantive results from the OLS estimation are mostly consistent with those of the 3SLS estimation. The detailed results can be obtained from the authors.
}

shopping budgets with which more items can be bought, resulting in a lower percentage of soft drink purchases or any one category.

\subsection{Cross-Subcategory Effects}

Table 2 summarizes the 3 SLS subcategory results. In the model for potato chips, only tortilla chips $(p<0.05)$ and cheese puffs $(p<0.05)$ are significant, i.e., the proportion of potato chip purchases is high when tortilla chip purchase proportion is low, but is high when cheese puffs purchases are high. Thus, potato chips are typically bought at the expense of tortilla chips (substitutes), but are bought together with cheese puffs (complements).

The tortilla chips purchases model indicates that potato chip purchases $(p<0.05)$, cheese puffs purchases $(p<0.05)$, pretzel purchases $(p<0.10)$, and the age of the store $(p<0.05)$ are significant predictors of tortilla chips purchases. Tortilla chip purchases take place at the expense of potato chips (substitutes), but they are high when purchases of cheese puffs and pretzels are high (complements). Furthermore, observed tortilla snack purchases are higher in newer stores.

In the corn chips model, only potato chip purchases $(p<0.10)$ and cheese puffs purchases $(p<0.05)$ are significant predictors of corn chips purchases. Corn chips purchases are low when purchases of potato chips are high (substitutes), but are high when purchases of cheese puffs are high (complements). In the cheese puffs model, purchases of potato chips $(p<0.01)$, tortilla chips $(p<0.05)$, and corn chips $(p<0.10)$ are the only significant predictor variables. Cheese puffs purchases are high when purchases of potato chips, tortilla chips, and corn chips are high, suggesting that cheese puffs are a complement with potato, tortilla, and corn chips. This result is consistent with those from other subcategories examined so far.

In the popcorn model, purchases of tortilla chips $(p<0.10)$ and pretzels $(p<0.001)$ are significant predictors of purchases of popcorn. Popcorn purchases are high when pretzel purchases are high (complements), but are low when tortilla chip purchases are high (substitutes). Among the control variables, store age $(p<0.05)$ and average household age $(p<0.05)$ have significantly negative effects on popcorn purchases. Older households shopping at older stores might purchase less popcorn than younger households because the product is primarily targeted at the younger generation, which tends to make more of its purchases at newer retail outlets.

Finally, in the pretzel model, tortilla chips purchases $(p<0.10)$, popcorn purchases $(p<0.01)$, age of store $(p<0.10)$, and average shopper age $(p<0.01)$ are the significant predictors. Pretzel purchases are high when purchases of tortilla chips are high (complements) and popcorn purchases are also high (complements). They also are high when the shopper's average age is high and when the store is old, 
Table 1 3SLS model results for salty snacks and soft drink purchases

\begin{tabular}{lll}
\hline Independent variable & $\begin{array}{l}\text { SSNACK model } \\
\text { Parameter estimate } \\
\text { (Standard error) (Hypothesis) }\end{array}$ & $\begin{array}{l}\text { BEV model } \\
\text { Parameter estimate } \\
\text { (Standard error) (Hypothesis) }\end{array}$ \\
\hline INTERCEPT & $-0.21(0.13)$ & $0.37(0.14)^{* *}$ \\
BEV & $0.62(0.23)^{* *}\left(\mathrm{H}_{1 \mathrm{a}}\right)$ & - \\
SSNACK & - & $1.37(0.94)\left(\mathrm{H}_{1 \mathrm{~b}}\right)$ \\
ADJ & $7.6 \times 10^{-3}\left(3.0 \times 10^{-3}\right)^{* *}\left(\mathrm{H}_{3 \mathrm{a}}\right)$ & $-0.01\left(6.7 \times 10^{-3}\right)\left(\mathrm{H}_{3 \mathrm{~b}}\right)$ \\
SSFT & $2.3 \times 10^{-5}\left(4.6 \times 10^{-5}\right)$ & - \\
BSFT & - & $-1.6 \times 10^{-2}$ \\
STRAGE & $-1.6 \times 10^{-5}\left(3.6 \times 10^{-5}\right)$ & $4.5 \times 10^{-6}\left(7.4 \times 10^{-5}\right)$ \\
SSIZE & $-1.8 \times 10^{-8}\left(2.3 \times 10^{-8}\right)$ & $3.0 \times 10^{-8}\left(4.3 \times 10^{-8}\right)$ \\
HHAGE & $3.6 \times 10^{-3}\left(2.0 \times 10^{-3}\right)^{* * *}$ & $-5.3 \times 10^{-3}\left(3.5 \times 10^{-3}\right)$ \\
HHINC & $2.1 \times 10^{-3}\left(7.8 \times 10^{-4}\right)^{* *}$ & $-3.0 \times 10^{-3}\left(2.0 \times 10^{-3}\right)^{* * *}$ \\
\hline
\end{tabular}

suggesting that pretzels are more popular with older households shopping at traditional outlets. ${ }^{8}$

The results from the subcategory models generally support $\mathrm{H}_{2}$. Some subcategories are complements while others are substitutes.

\subsection{Summary of Results}

Using store level data, we find asymmetric intrinsic and extrinsic cross-category effects. Purchases from the beverage category positively influence salty snack purchases (positive affinity or complements), but the converse is not true. Similarly, aisle adjacency of salty snack and soft drink beverage categories benefits salty snacks more than it does for soft drinks. Previous research, in the context of promotion-induced extrinsic cross-category effects, has also found such asymmetries [5, 18]. Their explanation is based on which category is generally bought as the primary category. Our results show that soft drinks may be the destination category that drives the purchases of salty snacks. Finally, some subcategories, such as potato chips, tortilla chips, corn chips, and popcorn, are intrinsic substitutes among themselves. However, each of these subcategories is intrinsically complementary with cheese puffs and pretzels.

The results on the asymmetric effects of salty snacks and soft drinks, taken together with those of Bezawada et al. [5], offer a nuanced understanding of cross-category effects between two complementary categories such as salty snacks and

\footnotetext{
${ }^{8}$ To validate the results from the system of subcategory models on the groups of complements and substitutes, we also performed cluster analyses (both k-means and Ward's hierarchical analyses) on the purchases of all salty snack subcategories. The cluster analyses produced two maximally differentiated clusters, one with potato, tortilla, corn chips, and popcorn, and the other with cheese puffs and pretzels. Thus, the results from the 3SLS models are consistent with those from the cluster analyses. Unlike cluster analysis, our approach provides the signs and magnitudes of the cross-effects.
}

beverages. They show a virtuous cycle of cross-category benefits for the retailer. Our results show that greater adjacency of the salty snacks and soft drinks aisles enhances the proportion of transactions in which salty snacks is purchased more than the proportion of transactions involving beverage purchases. This result is primarily because beverages are a destination category. However, once shoppers are in the store, if they buy chips, it triggers the psychological need to buy a soft drink such as cola [5]. This cueing results in greater purchases of cola when the aisles of chips and cola are more adjacent to each other [5]. Thus, the complementary categories salty snacks and soft drinks feed each other's sales.

\section{Discussion}

Our results are generalizable to many complementary food categories such as pasta and pasta sauce, bread and butter or jelly, meat and cheese, and waffle and syrup. In addition, they can be applied to other complementary categories such as movies and popcorn. In general, the findings should be relevant to contexts where two or more categories are consumed or potentially consumed during an occasion. An important caveat is application to business-to-business product categories such as office supplies and institutional purchase at warehouse clubs where our findings on asymmetry may have to be applied in a more nuanced fashion based on a careful study of the context and category usage.

Our results have important theoretical and managerial implications. From a theoretical perspective, the asymmetric intrinsic cross-category effects lend additional precision to the prevailing views of cross-category complementarity; significant intrinsic effects may occur in the absence of any marketing activities. Recall that our aggregate analysis across stores for a single grocery chain essentially removes temporal differences due to promotional activities. 
Table 2 Results for subcategory models

\begin{tabular}{|c|c|c|c|c|c|c|}
\hline \multirow[t]{2}{*}{ Independent variables } & \multicolumn{6}{|c|}{ Subcategory dependent variable } \\
\hline & Potato chips & Tortilla chips & Corn chips & Cheese puffs & Popcorn & Pretzels \\
\hline Intercept & $1.34(0.95)$ & $2.96(1.39)^{* *}$ & $0.27(0.33)$ & $-0.86(0.55)$ & $0.37(0.12)^{* * *}$ & $-1.02(0.37)^{* *}$ \\
\hline Beverage & $-0.49(1.38)$ & $-1.93(2.21)$ & $0.01(0.39)$ & $0.41(0.75)$ & $-0.36(0.17)$ & $0.99(0.62)$ \\
\hline Potato & - & $-1.61(0.73)^{* *}$ & $-0.28(0.16)^{*}$ & $0.57(0.21)^{* * *}$ & $-0.12(0.14)$ & $0.33(0.43)$ \\
\hline Tortilla & $-0.50(0.22)^{* *}$ & - & $-0.11(0.10)$ & $0.30(0.12)^{* *}$ & $-0.11(0.06)^{*}$ & $0.32(0.18)^{*}$ \\
\hline Corn & $-2.93(2.60)$ & $-3.93(5.25)$ & - & $1.68(0.91)^{*}$ & $-0.15(0.73)$ & $0.28(2.58)$ \\
\hline Cheese & $1.61(0.65)^{* *}$ & $3.03(1.35)^{* *}$ & $0.42(0.18)^{* *}$ & - & $0.31(0.23)$ & $-0.82(0.82)$ \\
\hline Popcorn & $-2.54(3.55)$ & $-6.71(4.71)$ & $-0.35(1.23)$ & $1.73(1.94)$ & - & $2.78(0.98)^{* * *}$ \\
\hline Pretzel & $1.25(0.99)$ & $2.81(1.38)^{*}$ & $0.24(0.38)$ & $-0.81(0.63)$ & $0.36(0.12)^{* * *}$ & - \\
\hline LINFT & $\begin{array}{l}2.2 \times 10^{-4} \\
\left(2.4 \times 10^{-4}\right)\end{array}$ & $\begin{array}{l}3.5 \times 10^{-4} \\
\left(3.8 \times 10^{-4}\right)\end{array}$ & $\begin{array}{l}6.9 \times 10^{-5} \\
\left(7.1 \times 10^{-5}\right)\end{array}$ & $\begin{array}{l}-1.3 \times 10^{-4} \\
\left(1.3 \times 10^{-4}\right)\end{array}$ & $\begin{array}{l}2.4 \times 10^{-5} \\
\left(3.2 \times 10^{-5}\right)\end{array}$ & $\begin{array}{l}-5.8 \times 10^{-5} \\
\left(1.1 \times 10^{-4}\right)\end{array}$ \\
\hline ADJ & $-3.4 \times 10^{-3}(0.01)$ & $-0.01(0.02)$ & $\begin{array}{l}-3.0 \times 10^{-4} \\
\left(3.2 \times 10^{-3}\right)\end{array}$ & $\begin{array}{l}2.6 \times 10^{-3} \\
(0.01)\end{array}$ & $\begin{array}{l}-1.8 \times 10^{-3} \\
\left(2.4 \times 10^{-3}\right)\end{array}$ & $\begin{array}{l}4.9 \times 10^{-3} \\
\left(7.3 \times 10^{-3}\right)\end{array}$ \\
\hline STRAGE & $\begin{array}{l}-3.2 \times 10^{-4} \\
\left(2.2 \times 10^{-4}\right)\end{array}$ & $\begin{array}{l}-6.9 \times 10^{-4} \\
\left(3.3 \times 10^{-4}\right)^{* *}\end{array}$ & $\begin{array}{l}-6.8 \times 10^{-5} \\
\left(7.8 \times 10^{-5}\right)\end{array}$ & $\begin{array}{l}2.1 \times 10^{-4} \\
\left(1.2 \times 10^{-4}\right)\end{array}$ & $\begin{array}{l}-8.6 \times 10^{-5} \\
\left(3.3 \times 10^{-5}\right)^{* *}\end{array}$ & $\begin{array}{l}2.3 \times 10^{-4} \\
\left(1.1 \times 10^{-4}\right)^{*}\end{array}$ \\
\hline SSIZE & $\begin{array}{l}-6.0 \times 10^{-8} \\
\left(8.4 \times 10^{-8}\right)\end{array}$ & $\begin{array}{l}-8.8 \times 10^{-8} \\
\left(1.5 \times 10^{-7}\right)\end{array}$ & $\begin{array}{l}-1.7 \times 10^{-8} \\
\left(2.4 \times 10^{-8}\right)\end{array}$ & $\begin{array}{l}3.3 \times 10^{-8} \\
\left(4.8 \times 10^{-8}\right)\end{array}$ & $\begin{array}{l}-5.2 \times 10^{-9} \\
\left(1.7 \times 10^{-8}\right)\end{array}$ & $\begin{array}{l}1.48 \times 10^{-8} \\
\left(5.0 \times 10^{-8}\right)\end{array}$ \\
\hline HHAGE & $-0.01(0.01)$ & $-0.03(0.02)$ & $\begin{array}{l}-2.2 \times 10^{-3} \\
\left(4.0 \times 10^{-3}\right)\end{array}$ & $\begin{array}{l}8.4 \times 10^{-3} \\
\left(7.1 \times 10^{-3}\right)\end{array}$ & $\begin{array}{l}-4.2 \times 10^{-3} \\
\left(1.5 \times 10^{-3}\right)^{* *}\end{array}$ & $\begin{array}{l}0.01 \\
\left(3.9 \times 10^{-3}\right) * * *\end{array}$ \\
\hline HHINC & $-0.01(0.01)$ & $-0.01(0.01)$ & $\begin{array}{l}-8.4 \times 10^{-4} \\
\left(1.8 \times 10^{-3}\right)\end{array}$ & $\begin{array}{l}3.0 \times 10^{-3} \\
\left(3.3 \times 10^{-3}\right)\end{array}$ & $\begin{array}{l}-1.5 \times 10^{-3} \\
\left(7.4 \times 10^{-3}\right)\end{array}$ & $\begin{array}{l}4.1 \times 10^{-3} \\
\left(2.0 \times 10^{-3}\right)\end{array}$ \\
\hline
\end{tabular}

${ }^{*} p=0.10$ (significant level); ${ }^{* *} p=0.05$ (significant level) ${ }^{* * *} p=0.01$ (significant level)

Although we do not study cross-category effects at the consumer level, the intrinsic asymmetries we find are consistent with differentially encoded memory representations. As noted earlier, product category knowledge can be represented as conceptual nodes linked into associative networks [20]. Over time and across varied usage occasions, shoppers learn to consume soft drinks with salty snacks, but with relatively few other categories. Consequently, they form only a few associative links with those other categories, but the association between soft drinks and salty snacks is strong. When soft drinks are encountered in the store, they act as a retrieval cue, and through a process of spreading activation [3], and then retrieval, the purchase of the strong-associated salty snack category concept is likely. Now, assume that shoppers have also learned to consume salty snacks with many other categories. In this case, they have a larger number of associations of varying strengths with those categories, such as hamburgers, salads, and beer. Therefore, because salty snacks are associated with many other products, in-store cueing when salty snacks are encountered is less likely to lead to retrieval of the soft drink product category [19]. The net result of such differential category representations would be the asymmetric cross-category effects reported by our results.

Although our results are for two categories, they could be applied and generalized to multiple categories. The intrinsic and extrinsic effects could be analyzed through similar models for each category and the cross-effects could be studied for similarities and asymmetries. For example, in the case of toothpaste, mouthwash, and toothbrush, we could find that the effects of toothpaste and mouthwash on toothbrush may be similar, but the effects of toothbrush on toothpaste may be different from those on mouthwash.

Our results have strong actionable implications for managers. From a managerial standpoint, the finding that crosscategory purchase effects and aisle adjacency effects are asymmetric can help both retailers and manufacturers. With the proliferation in categories, retailer strategies are becoming increasingly challenging [12]. Retailers interested in boosting the combined purchases of salty snacks, and of salty snacks and soft drinks, can focus on encouraging soft drinks purchases by stocking salty snacks close to soft drinks. Stocking decisions are important for efficient replenishment in the retail stores [9]. This arrangement would prompt increased consumption of both salty snacks and soft drinks. If, however, the retailers' goal is to increase beverage purchases, our results suggest that they may not gain as much by enhancing salty snack purchases.

Identifying the cross-demand patterns among categories and subcategories can provide useful managerial insights. The cross-demand patterns in our data appear in Fig. 1. 


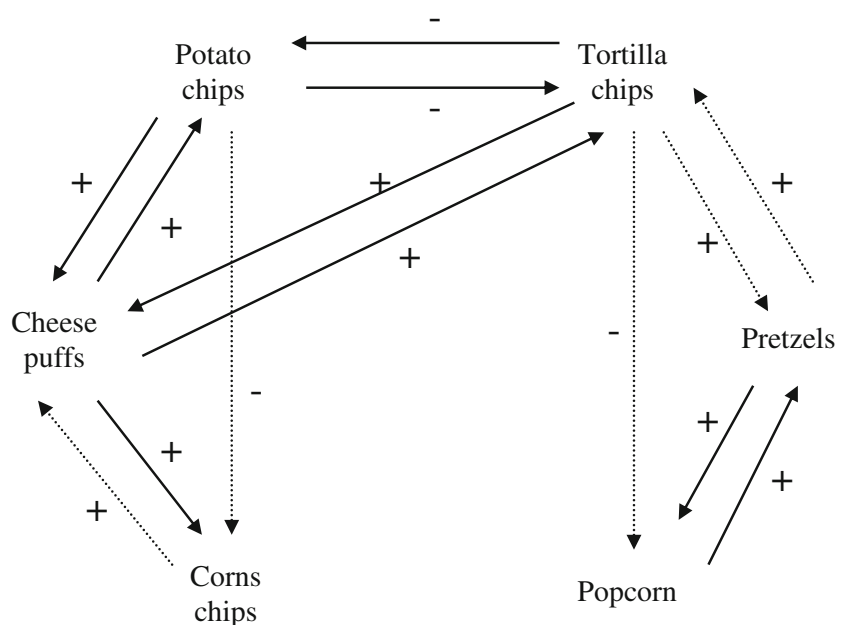

Fig. 1 Cross-demand patterns in salty snack subcategories snacks. Arrow points to dependent variable. All relationships significant at $p<0.05$ or better, except for dashed lines $(p<0.10)$

Knowing that some subcategories are substitutes while others are complements is managerially useful. Managers may want to identify the groups of subcategories that are distinct from one another (i.e., within the group), and the subcategories that are substitutes, but across the groups, they become complements. For example, the marketing manager of a manufacturer, like Frito Lay, who is interested in expanding overall purchases of all salty snack subcategories may decide to promote or bundle potato chips and cheese puffs together (complements), rather than promoting potato and tortilla chips together (substitutes). These useful managerial insights come from readily available, aggregate store level data, which can be easily analyzed.

Based on such arguments, we propose a managerial decision grid for two product categories with high- and lowmargin categories relative to each other (Fig. 2). The two principal dimensions on the grid are the type of crosscategory effects (intrinsic and extrinsic) and the nature of interrelationship between the two categories (substitutes and complements). The extrinsic effects are of two types, temporal (e.g., promotions and deals) and nontemporal (e.g., aisle adjacencies). The combination of these dimensions produces six possible situations, each corresponding to key decisions for the retailer.

Using our methodology, the retail manager can first identify the driver and the driven categories. The identification of
Fig. 2 Proposed framework for managing related product categories $\underline{\text { Proposed Framework for Managing Related Product Categories }}$

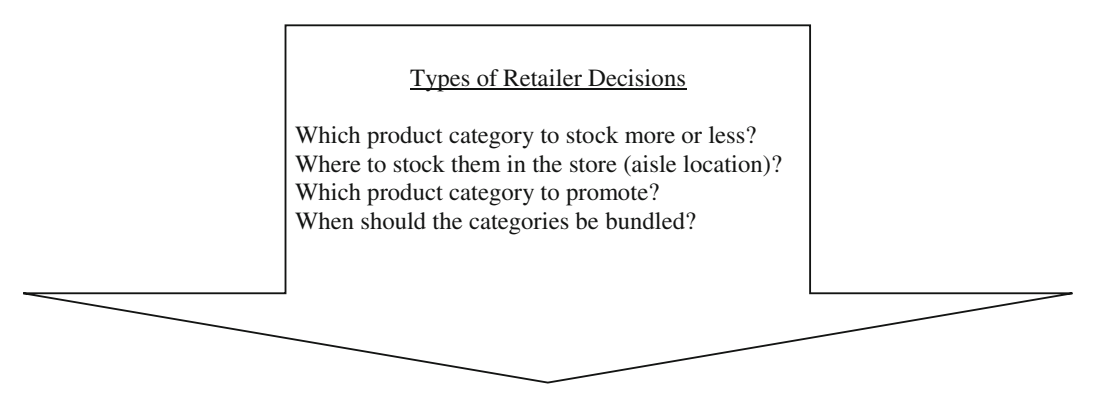

Suggested Marketing Actions for Two Product Categories, High-Margin and Low-Margin

Intrinsic Cross-Category Effect Extrinsic Cross-Category Effect

\begin{tabular}{|c|c|c|c|}
\hline & & $\begin{array}{c}\text { Temporal } \\
\text { (Promotion) }\end{array}$ & $\begin{array}{l}\text { Non-Temporal } \\
\text { (Aisle location) }\end{array}$ \\
\hline Substitutes & $\begin{array}{l}\text { Stock more of the } \\
\text { high-margin } \\
\text { category. }\end{array}$ & $\begin{array}{l}\text { Promote the } \\
\text { high-margin } \\
\text { category. }\end{array}$ & $\begin{array}{l}\text { Stock the two } \\
\text { categories apart. }\end{array}$ \\
\hline Complements & $\begin{array}{l}\text { Stock high levels of } \\
\text { both the categories. }\end{array}$ & $\begin{array}{l}\text { Promote the } \\
\text { driver category } \\
\text { (high/low } \\
\text { margin). If both } \\
\text { are drivers, } \\
\text { promote both } \\
\text { categories. }\end{array}$ & $\begin{array}{l}\text { Bundle the two } \\
\text { categories. } \\
\text { Stock the driven } \\
\text { category in two } \\
\text { aisles on either side } \\
\text { of the driver } \\
\text { category. }\end{array}$ \\
\hline
\end{tabular}


the driver and driven categories helps a retailer with stocking, promotion, and bundling decisions. For example, if a category is a driver category, a retailer should stock more of that category. The identification of the driver and driven categories is also important for managing cross-category pass-throughs [22].

Furthermore, based on the source of cross-category effects (intrinsic vs. extrinsic), managers can make better merchandising and inventory decisions. When the cross-category effect is intrinsic, the retailer should stock more of the highmargin category if the two categories are substitutes, but stock high levels of both the categories if they are complements.

When the cross-category effect is extrinsic, depending on whether the effect is temporal or not, and if the categories are substitutes or complements, managers have four possible response strategies. If the cross-category effect is temporal, and the categories are substitutes, it is effective to promote the high-margin category, while if the categories are complements, it is better to promote the driver category. ${ }^{9}$ When the cross-category effect is extrinsic and nontemporal, and the categories are substitutes, it is preferable to stock the categories far apart. Instead, complementary categories should be stocked together. Interestingly, this action may not be ideal from a consumer point of view because a consumer might like to have all the categories that satisfy a need to be presented together for easier selection. When the cross-category effect is extrinsic and nontemporal, and the categories are complements, bundling of the categories is the most appropriate decision. Furthermore, it may be effective for the retailer to stock the driven category in aisles on either side of the driver category.

The retailer's decision on the relative quantity of categories to stock will also depend on the margins of the driver and the driven categories as well as the sales per unit space for the categories. Typically, the driven category has a greater margin than that of the driver category, especially if the driver category is also a destination category, which is typically promoted to draw shoppers into the store. To maximize profits, retailers should stock the best combination of driver and driven categories based on the relative margins of the two categories in addition to the relative sales/square foot for the categories.

\section{Limitations and Future Research Directions}

Our research has certain limitations that future research could address. First, because the data are subject to aggregation bias,

\footnotetext{
${ }^{9}$ We realize that we do not analyze promotional data in this paper. However, because the expected effect of promotions is similar to that of nontemporal extrinsic effects, we are able to offer managerial suggestions on temporal intrinsic effects.
}

the results should be replicated and validated by a more detailed market basket analysis of cross-category and aisle adjacency effects. With the availability of time-series purchase data, such as market basket data, the extrinsic cross-category effects due to promotions can be studied. Moreover, unlike store level aggregate data, market basket data would allow us to analyze how often complementary categories are purchased in the same basket.

Second, our data were limited to two product categories. With data on additional categories, cross-category and aisle adjacency effects can be analyzed at a broader level. Third, the data were limited to one grocery chain. If data are available from additional chains, the analysis could be extended to study chain differences in cross-category and subcategory effects. Fourth, we treated aisle location as exogenous in our context of cross-store analysis. This assumption is reasonable because aisle adjacencies are not changed periodically within a store and they are somewhat fixed given the overall store layout, floor plan, and planogram. However, future research could explore the situation where aisle adjacency is treated as an endogenous variable. Fifth, we did not have data on category margins. With data on margins, we could generate additional insights on how to manage the driver and the driven categories.

Finally, some effects are not significant in our data, possibly due to sample size limitations. By applying our model and approach to a larger sample, we can explore if more effects become significant. Despite these limitations, we believe that using aggregate store data to model cross-category and aisle adjacency effects is a parsimonious and accessible analysis strategy that many managers and researchers will find useful.

Acknowledgments The authors thank Richard M. Durand for enabling data access and Gabriel Biehal for thoughtful discussions. They thank Frito Lay and an upstate New York retail chain for providing us with the data. Their thanks are also due to Laxminarayan Chakrapani for data assistance.

\section{References}

1. Ailawadi K, Beauchamp JP, Donthu N, Gauri D, Shankar V (2009) Customer experience management in retailing: communication and promotion. J Retail 85(1):42-55

2. Ainslie A, Rossi PE (1998) Similarities in choice behavior across product categories. Mark Sci 17(2):91-106

3. Anderson JR (1983) A spreading activation theory of memory. J Verbal Learn Verbal Behav 13(March):411-454

4. Bell DR, Lattin JM (1998) Grocery shopping behavior and consumer preference for retail price format: why large basket' shoppers prefer EDLP. Mark Sci 17(1):66-88

5. Bezawada R, Balachander S, Kannan PK, Shankar V (2009) Crosscategory effects of aisle and display placements: a spatial modeling approach and insights. J Mark 73(3):99-117

6. Bolton R, Shankar V (2003) An empirically driven taxonomy of retailer pricing and promotion strategies. J Retail 79(4):213-224 
7. Cohen JB, Basu K (1987) Alternative models of categorization: toward a contingent processing framework. J Consum Res 13(4 (March)):455-472

8. Divakar S, Ratchford BT, Shankar V (2005) CHAN4CAST: a multichannel multiregion forecasting model for consumer packaged goods. Mark Sci 24(3):333-350

9. Dong Y, Dresner M, Shankar V (2007) Efficient replenishment in the distribution channel. J Retail 83(3):253-278

10. Dreze X, Hoch SJ, Purk ME (1994) Shelf management and space elasticity. J Retail 70(4):301-326

11. Elrod T, Russell G, Shocker A, Andrews R, Bacon L, Bayus B, Carroll D, Johnson R, Kamakura W, Lenk P, Mazanec J, Rao V, Shankar V (2002) Inferring market structure from customer response to competing and complementary products. Mark Lett 13(3):219230

12. Gauri DK, Trivedi M, Grewal D (2008) Understanding the determinants of retail strategy: an empirical analysis. J Retail 84(3):256-267

13. Harlam BA, Lodish LM (1995) Modeling consumers' choices of multiple items. J Mark Res 32(November):392-403

14. Inman J, Shankar V, Ferraro R (2004) The roles of channel-category associations and geodemographics in channel patronage. J Mark 68(2):51-71

15. Iyer ES (1989) Unplanned purchasing: knowledge of shopping environment and time pressure. J Retail 65(1):40-57

16. Kahn BE, McAlister L (1997) Grocery revolution: the new focus on the consumer. Addison-Wesley, Reading

17. Kumar V, Shah D, Venkatesan R (2006) Managing retailer profitability: one customer at a time. J Retail 82(4):277-294

18. Manchanda P, Ansari A, Gupta S (1999) The 'shopping basket:' a model for multi-category purchase incidence decisions. Mark Sci 18(2):95-114

19. Meyers-Levy J (1989) The influence of a brand name's association set size and word frequency on brand memory. J Consum Res 16: 197-207

20. Mitchell AA, Dacin PA (1996) The assessment of alternative measures of consumer expertise. J Consum Res 23(December):219-239

21. Mulhern FJ, Leone RP (1991) Implicit price bundling of retail products: a multiproduct approach to maximizing store profitability. J Mark 55:63-76
22. Pancras J, Gauri DK, Talukdar D (2013) Loss leaders and crosscategory retailer pass-through: a Bayesian multilevel analysis. J Retail 89(2):140-157

23. Ratneshwar S, Shocker AD (1991) Substitution in use and the role of usage context in product category structures. J Mark Res 28(August): 281-295

24. Ratneshwar S, Pechmann C, Shocker AD (1996) Goal-derived categories and the antecedents of across-category consideration. J Consum Res 23(December):240-250

25. Russell GJ, Kamakura WA (1997) Modeling multiple category brand preference with household basket data. J Retail 73(Winter):439-461

26. Russell GJ, Petersen A (2000) Analysis of cross category dependence in market basket selection. J Retail 76(3):367-392

27. Russell GJ, Ratneshwar S, Shocker AD, Bell D, Bodapati A, Degeratu A, Hilderbrandt L, Kim N, Ramaswami S, Shankar V (1999) Multiple category decision-making: review and synthesis. Mark Lett 10(3):319-332

28. Shankar V (2011) Shopper marketing: current insights, emerging trends, and future directions. MSI Monograph: Relevant Knowledge Series

29. Shankar V (2014) "Shopper marketing 2.0: opportunities and challenges," Working Paper, Texas A\&M University, College Station

30. Shankar V, Bolton R (2004) An empirical analysis of determinants of retailer pricing strategy. Mark Sci 23(1):28-49

31. Shankar V, Inman J, Mantrala M, Kelly E, Rizley R (2011) Innovations in shopper marketing: current insights and future issues. J Retail 87(July):S29-S42

32. Sujan M, Dekleva C (1987) Product categorization and inference making: some implications for comparative advertising. J Consum Res 14(December):372-378

33. Supermarket News (1997, May 10) Acme pops top on soft drinks

34. Urban TL (1998) An inventory-theoretic approach to product assortment and shelf-space allocation. J Retail 74(1):15-35

35. van Heerde HJ, Leeflang PSH, Wittink DR (2000) The estimation of pre- and post promotion dips with store-level scanner data. J Mark Res 37(August):383-395

36. Zellner A (1962) An efficient method of estimating seemingly unrelated regression equations and tests for aggregation bias. J Am Stat Assoc 57:348-368 